\title{
Ignoranz, die Wissen erzeugt
}

\section{„Spezifiziertes Nichtwissen“ und Wissensersatzmechanismen im Spiegel des ungarischen Gesundheits- und Schulwesens}

\author{
Von Gábor Eröss
}

\begin{abstract}
„Ich vertraue den Daten nicht, denn ich war selber Arzt. “ (Führender ungarischer Gesundheitspolitiker)
\end{abstract}

\begin{abstract}
„,Politics finds its sources not only in power, but also in uncertainty [...] Governments not only 'power' [...]; they also puzzle. Policy-making is a form of collective puzzlement on society's behalf." (Heclo 1974: 305) ${ }^{1}$
\end{abstract}

\begin{abstract}
Zusammenfassung: In der Ära postbürokratischer Regulation, Transnationalisierung und evidenzbasierter Steuerung geht der Ruf nach Policy mit der Aufforderung nach Fakten einher. Wissen und kognitive Autorität legitimieren öffentliche Politiken. „Evidenz“ fehlt aber oft. Dieser Text untersucht sechs Evidenzsubstitutionsweisen: das transnationale, das sozialwissenschaftliche, das praktische, das charismatische, das lokale und das von Stakeholdern getragene Ersatzwissen, die in bestimmten Situationen die Wissenslücken im politischen Feld überbrücken. Wenn die alltägliche Funktionsweise oder die Legitimität der rational-legalen Herrschaft durch die langsame Konstruktion eines Ignoranzgefühls oder die plötzliche Erscheinung eines Ignoranzmoments geschädigt wird, muss das politische System Wissen von alternativen Quellen besorgen. Das Objekt meiner politischen Agnatologie und Wissensanthropologie ist also das gewusste Nichtwissen (oder „spezifische Ignoranz") in den öffentlichen Politiken, dessen Konstruktion und Inszenierung: die Substitution des mangelnden (institutionalisierten, formalen, wissenschaftlichen, bürokratischen, etc.) Wissens durch alternative Datenverarbeitungsweisen oder systemfremde (heteronome) Wissensformen, die, unter bestimmten Bedingungen, für bestimme Akteure, zuverlässiger, konkreter, oder, vor allem, authentischer und legitimer erscheinen. „Nichtwissen” trägt zur Emergenz eines postnationalen multilokalen Wissens bei.
\end{abstract}

\section{Eine Soziologie der politischen agnatology}

Das neue, angeblich dominierende Paradigma der evidence based policy klingt so: Politik muss sich mehr und mehr auf Tatsachen berufen. Das Problem ist „nur“, das „Evidenz“ - paradoxerweise - schwer zu finden und aufzuarbeiten ist:

„Es ist so, dass das Gesundheitswesen - wenn ich es sehr profan ausdrücken darf - in einem Gebäude wohnt, dessen Zimmer es nicht alle kennt. Nicht deswegen, weil es keine

1 Dieser Text ist eine erweiterte und vollkommen umgearbeitete Version von „Die Mobilisierung des Expertenwissens im öffentlichen Handeln. Historische Entwicklungen und Bedeutungen“, die auf der Tagung des Centre Interdisciplinaire d'Etudes et de Recherches sur l'Allemagne (CIERA) und des Instituts für Europäische Ethnologie der Humboldt Universität Berlin vorgetragen wurde (Berlin, 20.-21. Juni 2008).

Diese Analyse ist auf Basis des europäischen Projektes KNOWandPOL entstanden (Knowledge and Policy: The role of knowledge in the construction and regulation of health and education policy in Europe: convergences and specificities among nations and sectors. 6th Framework Program, koordiniert von Bernard Delvaux, Université Louvain-la-Neuve, Teilnehmer sind: Belgien, Deutschland, Frankreich, Norwegen, Portugal, Rumänien, Schottland und Ungarn). Ich leite eine ungarische Forschungsgruppe, die Gesundheitspolitiken analysiert. Siehe: www.knowandpol.eu.

Ich möchte mich für die Hilfe und den Rat von Hans-Peter Müller, Judit Gárdos und Márton Oblath bedanken.

Soziale Welt 60 (2009), S. $371-387$ 
technische Zeichnung oder keinen Ausführungsplan gibt, den man hinzuziehen könnte, sondern weil man keine Zeit dafür hat, man weiß nicht, in welcher Schublade er liegt, und wenn man ihn finden würde, wäre es nicht sicher, dass ihn jemand anschaut, der auch versteht, was er sieht. " (Führender Beamte des ungarischen Gesundheitsministeriums)

Die auf richtige, objektive Fakten basierende Politik ist eine Illusion, nicht nur weil die Fakten umstritten sind ${ }^{2}-$ auch in den Naturwissenschaften (Bechmann / Beck 2003), nicht nur weil die möglichen Folgerungen widersprüchlich, ${ }^{3}$ nicht nur weil die Wissenschaft ihr Monopol als Faktproduzent verloren hat, und nicht nur weil die Umsetzung und Übersetzung von „Fakten“ in „Politik“ nie direkt sein kann, sondern auch - und das könnte wohl ein spezifisches mitteleuropäisches Phänomen sein - weil es oft nicht einmal versucht wird, die öffentliche Politiken an formelle Fakten zu basieren:

„,Wir haben uns die erklärenden, einleitenden Texte der Gesetzesentwürfe angeschaut. Und wir sahen, dass zwei Drittel der Entwürfe überhaupt keine Informationen der Wirklichkeit betreffend erhielten. [...] So Passagen sind drinnen wie: das wird gut für die Leute, das wird nicht schlecht. In einem Drittel der Fälle gibt es wenigstens eine Tatsache, einen Trend, eine Schätzung, die etwas fundierter als eine Prophezeiung ist. [...] Wir können also sagen, dass die ungarischen Entscheidungsträger bei zwei Drittel der Fälle überhaupt nicht wissen, was die Konsequenz ihrer Entscheidung ist. [Frage: Diese Evidence Based Policy ist also nicht besonders kennzeichnend?] Nicht im Geringsten. Das ist kein ungarisches Phänomen. Wir haben einen Vergleich mit der Slowakei und mit Estland durchgeführt. In der Slowakei ist das die gleiche Situation, in Estland ist es besser. In West-Europa ist es viel besser. In Ost-Mittel-Europa ist es ungefähr so, wie bei uns. Die Politiker fühlen, dass sie allmächtig, allwissend sind, und das wollen sie durchsetzen. Sie brauchen keine Grübelei [um Entscheidungen zu treffen]. " (Leiter einer öffentlichen, aber unabhängigen Gesundheitsbehörde) $)^{4}$

Die Relevanz von „Nichtwissen“ als solches wurde in der sozialwissenschaftlichen Forschung in den letzten Jahren zunehmend (z.B. Wehling 2004, 2006, Gross 2007) festgestellt. Es umfasst für die meisten Spezialisten „Noch-Nicht-Wissen“, gewusstes und nicht-gewusstes „Nicht-Wissen-Können“, „Nicht-Wissen-Wollen“ und - für manche Autoren - auch falsches Wissen / Irrtümer oder Unsicherheit (Beck 1996: 302; Wehling 2004: 69ff; Geis 2008: 163).

Das Nichtwissen - wie ich es hier definiere - ist weder „Verwirrung“, noch „Unsicherheit“, weder „Irrtum“, noch die simple „Abwesenheit von Wissen“, sondern ein gewusstes Nichtwissen (Wehling 2006: 118ff) im Mertonschen Sinne: ,(A)s the history of thought, both great and small, attests specified ignorance is often a first step toward supplanting that ignorance with knowledge" (Merton 1968: 471; zitiert in: Wehling 2006: 62). Die Art von Nichtwissen, die ich hier analysiere, ist also jene, die - paradoxerweise - Wissen erzeugt, wenn auch Ersatzwissen. Nichtwissen ist also kein Antonym von Wissen, sondern eine Art ,verschobenes“ oder substituiertes Wissen. Ich schlage demnach eine Art ,politische Theorie des Nichtwissens“, eine political agnatology (R. Proctor, zitiert von Wehling 2006: 29) vor. Diese Art von Nichtwissen, diese ,spezifische Ignoranz“ entsteht dann, wenn die normalen, systemeigenen Wissensquellen des politischen Systems versiegen, oder die routinemäßigen Datenaufarbeitungsweisen nicht anwendbar sind; z. B.: der Staat kann mit statistischen Mitteln die er-

2 Wie John Law und andere Akteur-Netzwerk-Theoretiker hervorgehoben haben, gibt es in den meisten Forschungen einander widersprechende Teilergebnisse und viele nicht interpretierbare Daten (Law 2003).

3 Ein treffendes Zitat dazu: "Two reports, same evidence, and yet such different conclusions" (Marmot 2004: 906.).

4 Er ist auch Arzt und promovierter Jurist und hat seine juristische Doktorarbeit über dieses Thema geschrieben. 
wünschten Daten, Informationen nicht besorgen, und / oder kann sie mit bürokratischen Mitteln nicht aufarbeiten. Es wird also an systemfremde (heteronome) Wissensquellen und Interpretationsmuster appelliert, die die Ignoranz auflösen sollen.

Wenn entweder der alltägliche Funktionsprozess, oder die Legitimität der rational-legalen Herrschaft durch die langsame Konstruktion eines Ignoranzgefühls oder die plötzliche Erscheinung eines Ignoranzmoments geschädigt wird, muss das politische System Wissen von alternativen Quellen besorgen, um Funktionsstörungen entgegenzutreten oder seine Legitimität wiederherzustellen:

„,Nach Ansicht des Bundessozialgerichts verstößt die Beschränkung der finanziellen Unterstützung von derzeit 211 Euro [die jetzige Summe von Hartz-IV für Kinder] gegen das Grundgesetz. Diese Kürzung sei vom Gesetzgeber nicht ausreichend begründet und somit verfassungswidrig. [...] Ohne dass für Kinder der Bedarf ermittelt und definiert worden sei, habe der Gesetzgeber den Hartz-IV-Satz für Kinder um 40 Prozent gekürzt. "5

Es ist jedoch selten der Fall, dass dieses Moment der Ignoranz (,ohne dass für Kinder der Bedarf ermittelt und definiert worden sei“) und des Ersatzwissensbedarfes (,vom Gesetzgeber nicht ausreichend begründet") so klar vor unseren Augen erscheint.

Das Objekt meiner politischen Agnatologie ist also das gewusste Nichtwissen, oder „,spezifische Ignoranz" in der Politik: die Substitution (Ersatz) des mangelnden - institutionalisierten, formalen, wissenschaftlichen - Wissens durch andere Wissensformen, die - unter bestimmten Bedingungen, die soziologisch untersucht werden müssen - für bestimme Akteure zuverlässiger, „sicherer“ (z.B. lokales Wissen), verfügbarer, konkreter (z.B. Erfahrungswissen), legitimer (z.B. transnationale Wissensinstrumente) oder authentischer (z.B. charismatisch inszeniertes, „,verkörpertes“ Wissen) erscheinen. „Nichtwissen [stellt] nicht allein den vorgegebenen Ausgangspunkt (»Unwissenheit«) von Wissensbemühungen, sondern auch deren Folgen [dar]" (Wehling 2006: 31). Die Analyse von Nichtwissen zeigt, wie bestimmte Formen der Ignoranz bestimmte Formen des Wissens erzeugen. Um es wieder mit Merton zu sagen (der von der Wissenschaft spricht): Nichtwissen entlarvt ,long obscured pockets of ignorance as a prelude for newly focused inquiery" (Merton 1987: 8), und ,the specification of ignorance amounts to problem-finding as a prelude for problem-solving" (Merton 1987: 10)

Auf jeden Fall gibt es eine Spannung, einerseits zwischen einem Wissen, das immer komplexer, einem Noch-Nicht-Wissen, das immer größer wird, und anderseits der Rolle der für die jeweiligen zentralen Regierungen arbeitenden Experten, die ein eindeutiges, klares „Programm“, und ein zuverlässiges oder „,sicheres“ Wissen (reliable knowledge) müssen präsentieren können, das auch noch zusätzlich sozial „robust“ und „national legitim“ sein soll. Im „Mode 2“ ist es nicht die Wahrheit, sondern die Anwendbarkeit des Wissens, die zählt. Wenn dies klar wird, dann fangen die Experten selber an, entweder die Komplexität zu reduzieren und / oder „spezifiziertes Nichtwissen“" verschiedener Art zu ,verkaufen“.

Bei Wehling (2006) sind die drei Unterscheidungsdimensionen des Nichtwissens: Reflektivität (gewusstes oder ignoriertes Nichtwissen), „Intentionalität“ (absichtliches oder unabsichtliches Nichtwissen), und zeitliche „Stabilität“ (Dauerhaftigkeit oder Kurzlebigkeit) (Wehling 2006: 116ff; Geis 2008: 163). Aus den drei Dimensionen wähle ich je einen Pol aus: ich betrachte hier gewusstes, unabsichtliches und nicht dauerhaftes Nichtwissen. Denn es gibt in den hier von mir analysierten Fällen von Seiten der Entscheidungsträger einen „Willen zum Wissen“ (Foucault 2006) und - gerade deswegen - besteht die Ignoranz nicht dauerhaft. Es beinhaltet ein Streben nach Wissenssubstitutionsformen, die die Lücken überbrücken sollen.

5 Siehe http://www.zeit.de/online/2009/05/hartz-kinder-bundessozialgericht. 
„Nicht Wissen, sondern Nicht-Wissen ist das 'Medium' reflexiver Modernisierung“ (Beck 1996: 298). Für die Soziologie stehen „Nichtwissen und somit kaum handhabbare Unsicherheiten im Vordergrund“" (Kuhlicke 2006: 844). Dies ist besonders wahr für die politische Soziologie, und zwar gerade wegen dem eben genannten Rationalitätsversprechen.

In diesem Text präsentiere ich einige Hypothesen rund um die Paradoxie der spannungsreichen Beziehung zwischen „Wissen“ und „Policy“. Diese Beziehung wird hier nicht im Kontext einer hypothetischen Wissensgesellschaft (wo Wissen sich „ohne weiteres“ immer vermehrt) und auch nicht eines hypothetischen Zeitalters der evidenzbasierten öffentlichen Politik (wo Fakten sich fast ,von alleine“ den Politikern anbieten) beschrieben. „Die Fakten“, die die Entscheidungsträger ,,verwenden“ könnten, gibt es manchmal einfach gar nicht; daran leiden die Wissensbroker, die die fehlende Evidenz übermitteln sollen. Um diese Lücke auszugleichen, dieses gewusstes Nichtwissen zu bewältigen, werden verschiedene alternative Wissensformen hinzugezogen.

Die Ausgangsfrage lautet: Wie werden Wissenslücken als solche erstmals überhaupt identifiziert, also gewusst gemacht? Welche Arten von politischen Umständen sind besonders geeignet, ,gewusstes politisches Nichtwissen“, also eine Wissensnot, zu erzeugen?

Die beiden politischen Rahmenbedingungen, die einen zunehmenden Wissensbedarf erzeugen, sind die wirtschaftlich-gesellschaftliche „Krise“ und die politische Legitimationskrise. Das Gefühl einer „Gesellschaftskrise“, der aus ihr resultierende politische Reformdruck, die Idee der radikalen Veränderung des Kontextes, sind immer ein Aufruf zur Umstellung des Wissensrahmens, also zur erneuten wissenschaftlichen Sondierung der Bedingungen, zur möglichst vollständigen Abbildungen der „Realität“. Man sagt, die Wende (ein Speziellfall von Krise) sei in Ost-Mitteleuropa wie z.B. in Ungarn noch immer nicht vollendet, die Transition sei noch immer nicht hinter uns, das Schul- und Gesundheitssystem sei noch immer nicht Europa-kompatibel: neue Arten von Fachwissen und internationalen Fakten seien also sehr erwünscht. Auch die Legitimationskrise des westlichen Spätkapitalismus (Habermas 1973) und des osteuropäischen „Frühkapitalismus“ der letzten 20 Jahre haben dazu beigetragen, dass das Gewusste und das Nicht-Gewusste in der Politik neu definiert worden sind. Denn Legitimationslücken erzeugen Wissenslücken.

Auf den folgenden Seiten stehen einige Inszenierungsweisen des Nichtwissens und die Konstruktion der aus diesem stammenden Ersatzmechanismen im Mittelpunkt der Betrachtung.

Jede der folgenden sechs Abschnitte widmet sich einer bestimmten Form des Substitutionswissens: dem transnationalen, dem sozialwissenschaftlichem, dem praktischen, dem charismatischem, dem lokalen und dem von Stakeholdern getragenen Ersatzwissen, die allesamt in bestimmten Situationen die Wissenslücken im politischen Feld überbrücken. Zwei dieser Substitutionsformen werden von Experten, den - von Sozialwissenschaftlern meisterforschten - Wissensmaklern getragen (transnationales und soziologisches Ersatzwissen), zwei andere sind eingekörperte bzw. verkörperte Wissensformen (Erfahrungswissen und charismatisch inszeniertes Ersatzwissen), zwei entstehen durch eine Verschiebung der relevanten Ebene (transnationales bzw. lokales Ersatzwissen), und die letzte (die selber vielfältig ist) setzt eine Verschiebung des relevanten Feldes voraus (die Stakeholder bieten in der Wirtschaft, oder in anderen gesellschaftlichen Subsystemen erzeugtes Wissen an).

\section{Transnationales Ersatzwissen}

In den 80er und 90er Jahren ist eine doppelte Wende der öffentlichen Politik zu beobachten. Das erste Phänomen ist die Erscheinung postbürokratischer Regulationsformen (wie Evalua- 
tion, Qualitätsmanagement, incentives, capacity tools etc.), ${ }^{6}$ das andere die mit diesen eng verbundene Transnationalisierung. Über das ,post-bürokratische” Phänomen wird viel geforscht, die Politik verwandle sich demnach in „Governance“ (Altrichter / Brüsemeister / Wissinger 2007). Es wird behauptet, dass postbürokratische Regulationsformen vermehrt zur Policy-Verwendung von verschiedenen - eben transnationalen - Wissensformen führen, ja sogar zur Verwandlung von Wissen in Policy Instruments: das Wissen, besonders: internationale Evaluationen, rankings, benchmarking, so wie best practices ${ }^{7}$ werden zum „Policy Tool" (Delvaux / Mangez 2008).

Internationale Organisationen sehe ich hier nicht generell als Vertreter der umstrittenen Globalisierung, sondern als Institutionen, die - wie die OECD, die WHO, aber auch Eurostat - kontinuierliche Datenerhebungen fordern, und zwar aufgrund der Legitimationskrise des Staates (siehe oben), dessen Wissensinstrumente und traditionell gesammelten Daten delegitimiert werden (siehe PISA-Schock in Deutschland). In vielen Ländern - besonders in OstMitteleuropa - werden die aus den transnationalen Evaluationen entstehenden Vergleiche und die Consulting-Rhetorik zu einer neuen politischen Umgangssprache.

Best practice - das Wissen über erfolgreiche, oft weit entfernte Länder - wird auch viel zitiert, von den internationalen Organisationen, von Experten, die sich der sachverständigen Beratung widmen, oder von den nationalen Entscheidungsträgern selber:
„Bezüglich der Gesundheitsreform war der Ausgangspunkt, dass wir die besten europä- ischen Krankenversicherungstypen finden. Über die persönliche Erfahrungssammlung hin- aus versuchten wir natürlich die Krankenversicherungen all jener Staaten anzuschauen, deren Praxis von uns übernommen werden könnte. Gründlich schauten wir die französi- schen, österreichischen, deutschen, belgischen Krankenversicherungssysteme durch, die auf klassischen Bismarckschen Prinzipien ruhen. Wir haben eine internationale Konferenz organisiert, viele unserer Experten arbeiten bei verschiedenen internationalen Organisa- tionen, angefangen von der OECD über die Weltbank bis zur WHO. Wir baten sie um Studien. Das gesundheitspolitische, krankenversicherungspolitische Programm der sozia- listischen Partei beruhte darauf. " (Ungarische Fachpolitikerin, ehem. Staatssekretärin)

Auch wenn wir (vorübergehend) annehmen, dass durch diese neuen, postbürokratischen Steuerung- und Wissensformen das vorhandene Wissen sich immer vermehrt, stellen diese transnationalen Wissensformen natürlich sofort die Frage der Relevanz dekontextualisierter Methoden, Daten und Konzepte. ${ }^{8}$ Hier geht es aber weder um eine Hymne an das transnationale Wissen, noch um seine Kritik, sondern um die Beschreibung der historischen Emergenz, der sozialen Konstruktion, und der politischen Legitimation von diesen „Wissensprodukten“ als Ersatzwissensformen der bürokratischen, nationalen und disziplinären Wissens-, bzw. Wissenschaftsformen. Wie kam es dazu, dass PISA in Deutschland - oder in Ungarn - plötzlich als eine Ersatzform des mangelnden Wissens über Ungleichheiten (die Segregation der Schüler mit Migrationshintergrund bzw. der Roma-Schüler) inszeniert worden ist?

6 Incentives und capacity tools bedeuten Wissen über die potenziell effizienten Motivationen und Werkzeuge.

7 Wie zum Beispiel die „guten Schüler“, wie Finnland und Schweden in der Schulpolitik, oder - für ungarische liberale Experten die Niederlande in der Gesundheitspolitik. (In Ungarn sind es die mit den Sozialisten zusammenregierenden Liberalen, die das holländische Gesundheitssystem - der Privatversicherungen - ,importieren“ wollten. Sie haben sogar den ehemaligen niederländischen Gesundheitsminister zum Hauptberater des ung. Ministeriums berufen).

8 Viele Vergleiche, besonders die Rankings, werden kritisiert, dass eine riesige Diskrepanz zwischen ihnen und den subjektiven Einschätzungen und der eigentlichen Zufriedenheit der Patienten besteht, was die Gesundheitssysteme betrifft (Blendon / Minah / Benson 2001). Auch Benchmarking wird als unzuverlässige Regulierungsmethode betrachtet (Arrowsmith / Sisson / Marginson 2004). 
Was die Legitimitätsfrage angeht, parallel zu den eventuellen Gewinnen an Legitimität durch OECD in manchen Ländern, können andere - unter bestimmten Bedingungen, die empirisch geforscht werden sollen - mehr oder weniger ,immun“ bleiben; wenn zum Beispiel das Internationale per se politisch und / oder methodologisch (wissenschaftlich) verdächtig ist, wie im Falle von PISA in Frankreich (Grenet 2008). Entscheidungen werden u.a. entpolitisiert und so gegen Kritik immunisiert (Voßkuhle 2008 a: 12ff; Schuppert 2008: $290 \mathrm{f}$ ), was zu einem Legitimationsdilemma führt (Schuppert 2008: 273; Weingart: 2003: 91 f), sagen die Kritiker, nicht nur in Frankreich. Andere Wissenssubstitutionsformen gibt es aber auch...

\section{Soziologisches Ersatzwissen}

Wenn ein Mangel an Fakten, an anwendbaren wissenschaftlichen Ergebnissen identifiziert wird, wird immer versucht, die Lücken durch Fakten anderer Art zu überbrücken. Warum, wie, und unter welchen Bedingungen (wenn überhaupt!) konstruieren Soziologen - denen ich hier eine zunehmende Rolle zuschreibe - die kognitive Autorität, die eine demokratische Wissensnutzung legitim macht?

Die Soziologie hat eine schizophrene Rolle inne: sie kritisiert die Consulting-Rhetorik, und zwar grundsätzlich, profitiert aber auch gleichzeitig von der Unsicherheit anderer Experten und von den Legitimationsbedürfnissen der Entscheidungsträger. Sie ist da, (a) wenn die offiziellen Daten nicht zuverlässig sind, (b) statt nationalen Daten Wissen über lokale Phänomene benötigt wird, (c) ein Paradigmenwechsel der bisherigen Policy verlangt wird, (d) (neue) Zielgruppen zu bestimmen sind (Targeting).

Targeting - Wissen und Policies Zielgruppen betreffend - ist ein Mechanismus, der transnationale protowissenschaftliche Kategorien - die hauptsächlich die Soziologie erfunden hat - zu öffentlich-politischen und diese in lokal-institutionelle Kategorien verwandelt. Sehr interessant ist dabei, welche Repräsentationen - Kategorien und Bilder - der immer-zu-reformierenden gesellschaftlichen Sub-Systeme durch Soziologen-Experten produziert werden und wie die Konkurrenz zwischen den verschiedenen Disziplinen und den verschiedenen Beiträgen unterschiedlicher Wissensproduzenten in die politischen Repräsentationen eingebaut wird.

Nehmen wir das Beispiel der (De)Segregation der Roma im ungarischen Schulsystem. Seit 1993, als Konsequenz der neuen Gesetzgebung über die so genannten nationalen und ethnischen Minderheiten und der wiederholten Warnungen der Ombudsmänner für Minderheiten und für Datenschutz, sind flächendeckende Datenerhebungen im Bereich ethnischer Hintergrund verboten. (Eröss et al. 2007: 61). Das aus einer Menschenrecht- und Datenschutzperspektive völlig legitime Verbot führt zu einer statistischen Wüste. Dass das keine exotische (ungarische) Tatsache ist, zeigt auch die deutsche Lage, wo „Schüler mit Migrationshintergrund“" zwar als eine sozialwissenschaftliche und - viel mehr - als eine politische Kategorie existieren, aber nicht als statistische Kategorie, da viele dieser Kinder als Deutsche und auch viele als Einwanderer (Nicht-Staatsbürger) registriert sind. Auch in Frankreich führt diese Situation zu verzweifelten (wenn auch originellen und innovativen) Lösungen. Wie der von Felouzis und seinen Mitarbeitern gezeigte Weg: diese haben nämlich die Schüler mit ausländisch klingelnden Vornamen gezählt, in verschiedene Ländergruppen (Nordafrika, Türkei, etc.) eingeordnet (Felouzis et al. 2005) und mit diesen Daten die bisher versteckten ethnischen Segregationsmuster festgestellt. Und zwar da, wo die - den Politiker - gewohnten Wissensproduktionsmechanismen versagen.

Targeting ist also der Hauptzugang für Sozialwissenschaftler in die Policy-Making. In Ungarn geschah das so:

„,Der Begriff der Segregation ist auf Roma-Kinder bezogen, aber da die Identität frei wählbar ist, und von den 800 Tausend Roma weniger als 200 Tausend sich dazu bekannt haben, 
kann man sie nicht als Zielgruppe definieren, weder bei der Gesetzgebung, noch bei der Ausarbeitung von Programmen oder vor der EU. [...] Und wir dachten, nach einer Idee von [dem Soziologen] Gábor Havas, dass da die Gruppe der Eltern ohne Grundschulabschluss [in Ungarn: 8 Schuljahre] über $80 \%$ der Roma einschließt, wir diese Gruppe "sozial benachteiligt « nennen [und sie als Zielgruppe definieren]. "(Viktória Mohácsi, ehem. Kommissarin für die Integration von Roma und sozialbenachteiligten Schülern).

Targeting kann aber wiederum oft zu Nichtwissen führen (nicht alle Ersatzwissensformen konstituieren ein „ultimatives “Wissen); denn ein effektives Targeting setzt genaue Daten über die Zielgruppe voraus, die oft nicht vorhanden sind, weil die Übertragung von Wissen vom lokalen zur nationalen Ebene einen mehr oder weniger großen Verlust an Information verursacht:

„Ich sprach mit einem Notar eines Dorfes, der zynisch bemerkt hatte, dass er im Flur des Amtes ausgehängt hatte, dass diejenigen, die wollen, eine Erklärung über den Sozialbenachteiligten-Status abgeben können. [..] Es ist also ein System gegeben, in dem alle Regeln auf die Kategorie der mehrfach sozial Benachteiligten basieren, aber es gibt keine verlässlichen Daten, wie viele in diese Kategorie gehören. [...] Die Ortschaft Sárkeresztúr, eine typische Ghettoortschaft [mit überwiegender Roma-Bevölkerung], hat nicht die nötige Unterstützung bekommen [bei einer Ausschreibung], weil sich drei Ortschaften zusammengeschlossen haben und die anderen zwei falsche Daten über die geplanten Investitionen eingereicht haben. Fertig, Sárkeresztúr ist durch." (Havas 2009)

Die Soziologie - die von Chancen(un)gleichheiten spricht - hat das Privileg, im Prozess der Beschreibung der sozialen Realität sich als selbstverständlich kompetent darstellen zu können, weil sie „Die“ Wissenschaft der Gesellschaft ist. In Zusammenhang mit der Segregation von Roma (und anderen sozial benachteiligten Gruppen) wurde PISA in Ungarn weniger als eine Datenquelle als ein Signal wahrgenommen: das ungarische Schulsystem sei ineffizient und ungerecht; und zwar ineffizient, weil ungerecht. ${ }^{9}$ Durch diese neue kausale Kette wird die kognitive Autorität der Segregationsforscher, also der Soziologen, legitimiert, in dem es nicht mehr nur um die Roma, sondern um die ganze Gesellschaft geht: „The main idea is that investing extra public money into Roma education would pay off even in fiscal terms. [...]. The motivating idea behind our analysis is the notion that investing into somebody's education will lead to benefits not only to the person in question but also to the whole society" (Kertesi / Kézdi 2006: 9). Im ungarischen Bildungswesen bestimmen jetzt die - von sozialwissenschaftlichen Experten überzeugten und informierten - Politiker, wie viele ,sozial schwache“ Schüler in den einzelnen Schulbezirken sein dürfen, wie sie positiv diskriminiert werden sollen usw. Wissen über die Gesellschaft und deren Subsysteme wird in der Sozialwissenschaft konstruiert, im politisch-wissenschaftlichen Spannungsfeld dekonstruiert und immer wieder übersetzt (Translation) bis es zur Kategorie und zur Zahl, zur „Evidenz“, und zur Rechtfertigung wird.

Nicht nur in Ungarn, und nicht nur im Schulwesen wurden die von den Sozialwissenschaftlern angebotenen Perspektiven in den letzten Jahren von Politikern aufgegriffen: „The topic of inequalities in health was unpopular in Britain in the $1980 \mathrm{~s}$. An impressive review of evidence was insufficient to convince a government to act. A change of government in the $1990 \mathrm{~s}$ meant that government was willing to take action on health inequalities. A review of the scientific evidence and accompanying policy recommendations were sufficient for a government to implement many of them." (Marmot 2004: 906).

In Ungarn wurden im Jahre 2006 äußerst umstrittene, radikale Gesundheitsreformen begonnen. Diese sind auf der Basis eines „White Books“( (beziehungsweise eines grünen Buches),

9 Siehe sämtliche englischsprachige Dokumente unter: http://oecd-pisa.hu/english/publications.php. 
das fundamentale Fakten und mögliche Policy-Richtungen auflistet, zu verwirklichen. (Eröss / Mund et al. 2007: 24), und bezieht sich vor allem auf Fakten der sozialen und räumlichen Ungleichheiten, die das Land charakterisieren, also auf soziologische Argumente. Es ist ein Versuch, soziales und soziogeographisches Wissen, das eine steigende kognitive Autorität besitzt, als Legitimationsfaktor des Reformkurses einzusetzen.

Wenn die von sozialwissenschaftlichen Experten (incl. sozial „empfindlichen“ Wirtschaftwissenschaftlern) betriebene Soziologisierung ${ }^{10}$ in Ungarn dominant wird, dann können wir dieses Phänomen als Paradigmenwechsel im Wissensverbrauch des Gesundheitswesens beschreiben: statt des wissenschaftlich-medizinischen Wissens über das eigene Feld und des auf dieses basierende öffentliche Gesundheitspolitiken herrscht „,fremde“ Expertise und „fremde“ Perspektive, und zwar die der Sozialwissenschaften. ${ }^{11}$ Die oben genannten Gründe (die wirtschaftlich-gesellschaftliche Krise und die politische Legitimationskrise) haben dazu geführt, dass „Policies“ immer mehr durch sozialwissenschaftliches (oder sozialwissenschaftlich aussehendes) ${ }^{12}$ Expertenwissen begründet oder legitimiert werden (Eröss / Mund et al. 2007; Bajomi / Berényi / Neumann 2007).

\section{Lokales Ersatzwissen}

Auf welcher Grundlage stehen also die öffentlichen Politiken? Policies sind immer weniger das Ergebnis von staatlichen und immer mehr das von sub-nationalen bzw. lokalen und internationalen Einflüssen. Der Staat, kleinstädtische Behörden, transnationale Akteure und all ihre Experten interagieren an der Ebene der Perspektiven, Schemata, Daten, Kategorien und Konzepte, die für die Beschreibung von bestimmten sozialen Systemen (wie Erziehung und Gesundheit) gebraucht werden, und kämpfen um die Anerkennung der Relevanz der eigenen Perspektive, der eigenen Daten und Kategorien - und dadurch für die eigene Legitimität.

Das heißt, dass eine Art von Nicht-Wissen auch dadurch entsteht, dass das benötigte Wissen nicht dort vorhanden ist, wo die Entscheidungen getroffen werden. Die Entkopplung von Wissensproduktionsort und Wissensnutzungsort führt entweder zum Nicht-Wissen pur, also der Abwesenheit von Wissen (Wehling 2006) oder zur Verwendung von lokal bzw. global erzeugten Wissensformen, die aber nicht unbedingt ohne Schaden den Informationsübertragungsprozess durchhalten, wie wir es oben beim Thema „Targeting“ gesehen haben.

Die globale Ebene haben wir oben auch schon erwähnt, jetzt kommen wir zur lokalen. Und zwar zur lokalen Ebene, die im dezentralisierten politischen System gleichzeitig Ort des Wissens und der Entscheidung ist. Ungarn und Zentral-Europa sind ein Laboratorium für eine Analyse der post-staatlichen Gesellschaft: Wo in West-Europa noch die Illusion (aber auch die Realität) einer integrierten Gesellschaft (im Sinne von Durkheim) vom Staat aufrechterhalten wird, sind es in Ungarn transnationale Einflüsse und die Street Level Bureaucracy (Lipsky 1984), das heißt, lokale Gemeindeverwaltungen oder gar die Institutionen selber (Krankenhäuser und Schulen), die über Gesundheit und Schulkarriere entscheiden und nicht einfach Gesetze implementieren. Die Spannung, die in Form von gegenseitigen „Nicht-Wissen"-Vorwürfen niederschlägt, ist aber unabwendbar, denn nicht nur die zentralen Experten werfen den Gemeinden vor, Informationen zu verschweigen, oder gar nicht zu sammeln, wie wir oben gesehen haben, auch die lokalen Akteure (street level bureaucracy) sind oft davon

10 Sozialwissenschaftler sprechen von sozialen, räumlichen und alterbedingten Ungleichheiten des öffentlichen Gesundheitsversorgungssystems.

11 Dass gleichzeitig auch eine Ökonomisierung stattfindet, will ich hier nicht analysieren.

12 Politisches Marketing benutzt auch sozialwissenschaftliche Methoden (z.B. Zielgruppen bestimmen und mit der Methode der Fokusgruppe testen), die ihren Wert in der politischen Arena erhalten. 
überzeugt, dass der Staat die lokalen Sachverhalte nicht versteht, bzw. nicht verstehen kann. In unserem bildungspolitischen Beispielfall war das genauso:

„,dieser Institutsleiter [Einschulungskommission] auf Komitatebene kennt ungefähr alle Schulen im Komitat. Und er weiß, wie die Leitung der einzelnen Schulen ist, und ob das Geld, das vom Ministerium für die Einstellung von Sozialpädagogen zur Verfügung gestellt wird, wirklich dafür verwendet wird. Es gibt Schulen, die das Geld erhalten, aber nichts für die Kinder tun. Die Antwort der Einschulungskommission ist, dass sie [in den Schulen, die schwindeln] weniger staatlich unterstützte Kinder mit speziellen Erziehungsbedürfnissen bestimmen. Also wir erreichen, dass die Schule nicht so viel Geld bekommt. Denn das Geld kommt nicht den Kindern zu Gute.

So werden als ungültig betrachtete Zahlen, Indikatoren, Statistiken über Zielgruppen des Staates (z.B. sozial benachteiligte Schüler) im lokalen Wissens- und Machtkontext durch andere, „gültigere" Zahlen ersetzt.

Ich fasse Kindergärten, Schulen, Erziehungsberatungsstellen und verschiedene Organe der Gemeindeverwaltung als sinnbehaftete Institutionen auf, die Teil des Netzwerkes der gegenseitigen Sinngebungen sind, und deren Aufgabe gleichzeitig die Konstruktion und Kontrolle der Bedeutungen und Kategorien, also von Form und Inhalt von lokalem Wissens ist (Eröss 2008 b). Dies bedeutet einerseits die lokale Deutung ,zentraler“ Kategorien (,sozial Benachteiligte“, „Legastheniker"), andererseits die Konstruktion lokaler Kategorien (,gutes“ Gymnasium; Eliteschule; ein Junge, der in eine Hauptschule gehört; Roma-Kinder, die ein „Sozialisationsdefizit" haben etc.). ${ }^{13}$ Bedeutung wird lokal durch Rituale gefestigt (Wulf et al. 2006); wir gehen davon aus, dass die starke Autonomie der lokalen Akteure zu einem Formenreichtum von diesen Ritualen und Kategorien führt. Das heißt: radikal gesagt, kann gar kein globales oder nationales Wissensmuster die lokalen Realitäten hervorbringen. Unterschiede sind nämlich keine sich von einem bestimmten oder bestimmbaren Muster zufällig abweichende Formen, sondern Formen der Kontingenz im Sinne von Luhmann (Luhmann 1984: 152); sie sind also alle möglich, innerhalb eines sozialen Systems auffindbar, aber unvorhersehbar und voneinander unabhängig (Eröss 2008 a).

Ganz so, als ob sie das verstanden hätten, geben heutzutage paradoxerweise auch globale Institutionen Aufträge für lokale Analysen: auch die OECD gab einer der Landesrepräsentantinnen Ungarns den Auftrag, eine Studie auf lokaler Ebene durchzuführen, um die realen Wirkungen von nationalen Policies, die realen Bedeutungen von internationalen Kategorien und die realen Zahlen zu untersuchen, die sich hinter offiziellen Statistiken verbergen. Die in einem armen Komitat Nordungarns durchgeführte Studie hat gezeigt, wie entfernt die lokale Lage einerseits, die nationalen Zahlen und internationalen Kategorien anderseits voneinander liegen (Csányi 2007).

\section{Charismatisches Ersatzwissen}

Fachpolitik wird nicht nur durch Wissenschaft und Expertise, sondern auch durch die Verkörperung eines Ideals legitimiert, dadurch dass - etwa im Sinne einer Phänomenologie des Leibes - der Körper als die „Grundausstattung jedes Zugangs zur Welt“, „vorrangige Quelle des Wissenserwerbs, also als Träger von Erkenntnis“ (Hirschauer 2008: 997) gewürdigt wird.

13 Solche Kategorisierungen sind natürlich keineswegs nur symbolische Gesten: Street-level Bürokraten, die Schulverwaltung oder auch einfache Erziehungsberater haben verschiedene ihnen vom Gesetz verliehene Möglichkeiten, die sie nicht bloß zu einem Spiel der Bedeutungen befähigen, sondern zu einschneidenden Entscheidungen über lange Lebensabschnitte, ja sogar vielleicht über ganze Leben. 
Idealtypisch brauchen öffentliche Politiken im Kontext der legal-rationalen Herrschaft eine rationale, wissenschaftliche Begründung. Wenn es aber in einer modernen Gesellschaft an Legitimität legal-rationaler Natur und an Wissen rationaler-formalisierter Natur mangelt, wird an charismatische Legitimität appelliert, die durch eingekörperten Wissensformen verschiedener Art unterstützt werden. Ich postuliere also eine Analogie zwischen verschiedenen Herrschaftstypen und den zu denen gebrauchten Wissensformen.

Der Sportminister soll - in den meisten Staaten - am besten ein ehemaliger Sportler sein, um ein Vorbild im Weberischen Sinne zu sein, die Frauenministerin eben eine Frau. Der Körper weiß es: er ist ein Beweis. Auch in Deutschland scheint Familienministerin von der Leyen zu dieser Kategorie der Politiker zu gehören, die über ein unhinterfragbares charismatisches Wissen verfügen. Die Amerikaner hatten bei den Präsidentschaftswahlen 2008 den Wahl zwischen zwei Subtypen Weberscher charismatischer Legitimitätsanspruche: die des Helden (McCain) und die des Vorbildes (Obama); der eine und der andere verkörperte ein Erfahrungswissen, das auch politisch thematisiert wurde: die leibhaftigen Leiden des jungen McCain, die zu seiner Ablehnung von Folter geführt haben, und die afroamerikanischen Wurzeln, aber auch die Erfahrungen als Community Organiser (Sozialarbeiter), die den Politiker Obama geprägt haben.

Die Aufgabe der Desegregation der Roma-Kinder in Ungarn wurde durch die Person von Viktória Mohácsi repräsentiert. Die junge Journalistin und Aktivistin, selber eine Roma, wurde plötzlich 2002 zu einer der mächtigsten Politikerinnen des Landes, zur Kommissarin für die Integration von Roma und sozialbenachteiligten Schülern, zu einer Art Sonderministerin (Rechenschaft nur dem Minister schuldig). Sie analysiert, einige Jahre später in 2008, als sie schon lange nicht mehr im Ministerium, sondern als Europaabgeordnete tätig ist:

„,Und als ich ins Bild kam und Bálint Magyar [der damalige Bildungsminister] mir das Angebot machte, dann tat er das aus Gründen der Rasse [sic!] und der Chancengleichheit: er suchte jemanden, eine Frau, die jung ist und gut aussieht, und vor Kameras sprechen kann [...] Er bestand darauf, dass er auf jeden Fall, auch wenn es keine Frau ist, einen Zigeuner möchte, dem man es auch ansieht, denn er wollte ihn in der Öffentlichkeit agieren lassen."

Die Narrative einer ihrer Kolleginnen ist ähnlich. Man habe jemanden gebraucht, der mit Talent das Wissen der Experten (Soziologen) und den Willen der Entscheidungsträger (der Minister) verkörpere:

„, Gábor Kertesi, Gábor Havas und István Kemény [drei Soziologen] haben beschlossen, Bálint Magyar [den Bildungsminister] zu überzeugen, das, was sie seit Jahrzehnten forschen, und von dem sie wissen, was zu tun ist, umzusetzen. Diese drei-vier Menschen. Das schaut normalerweise so aus, dass, da der Kertesi den Minister gut kennt, er ihm eine EMail schreibt, er soll sie empfangen, denn sie haben einen Plan. Dieser Plan war, dass eine Organisation gegründet werden sollte, die einen nationalen, starken Wirkungskreis hat, starke Kompetenzen, und sie soll beginnen, den Gemeindeverwaltungen und Schulen zu vermitteln, effizienter bzgl. der sozial benachteiligten und Roma-Kinder zu sein. [...] Sie soll also den ganzen Staat umfassen und stark sein. [...] Dann sagte der Minister, sie sollen das auf maximal drei Seiten niederschreiben. [...] Und währenddessen kam der Auftrag von ganz oben, dass man in den Ministerien Zigeuner einstellen sollte, in hohen Positionen, auf der Höhe der ministerialen Kommissare. Die Vorstellung war, dass es dadurch besser wird, dass die Angelegenheit der Roma durch junge intellektuelle Roma mindestens auf Staatssekretärebene vertreten wird. [...] Viktória Mohácsi, die Roma ist, sollte ernannt werden. [...] Man sagte, der / diejenige sollte Zigeuner sein, deswegen kam keine andere in Frage. [Viktória Mohácsi] führte die beiden Begriffe in das Program ein, Integration 
und Desegregation. Und dann holte sie tief Luft und verkündete, ihr Program sei die Desegregation. " (Hochrangiger Beamte im Bildungswesen)

Das Wissen, das man braucht, ist in der Lebenserfahrung der ungarischen Roma als Gemeinschaft im Allgemeinen vorhanden, und wurde von damals an durch die Person von Mohácsi verkörpert. Die Experten, die die Desegregationspolitik der Regierung und Mohácsi mit Daten unterstützten und unterstützen, sind diejenigen, die schon seit Jahrzehnten dieses Thema erforschen. Mohácsi behauptet nicht, dass sie alles wisse, sie wird aber durch eine Art charismatische Legitimität verstärkt. Das ist kein Erfahrungswissen im engsten Sinne, sie sei selber nicht segregiert worden in der Schule (sie saß bloß in der letzten Reihe), metonymisch (pars pro toto) repräsentiert sie jedoch all die, die aus ethnischen Gründen ausgegrenzt sind.

\section{Praktisches Ersatzwissen}

Der Einsatz des praktischen Wissens oder Erfahrungswissens (Reichert et al. 2000) wird von Entscheidungsträgern selber als eine Übersetzung (Translation) dargestellt, die das außerpolitische Wissen als fachpolitisches Wissen in das politische Feld einbezieht:

„,Unsere Tätigkeit würde ich am ehesten mit der eines Übersetzungsbüros vergleichen. Die Angestellten des Ministeriums, die Beamten mit Arztausbildung oder die uns unterstützenden Hintergrundorganisationen können hervorragend definieren, was in der Gesundheitsbranche so im Allgemeinen gebraucht wird. "(Führender Beamte des ung. Gesundheitsministeriums)

Worauf wird man sich also stützen, wenn man keine Daten, keine wissenschaftliche Evidenz hat? Die Erfahrung der Machthaber selber - ob Street Level Bureaucrats oder Politiker - wird angewandt. Dies ist jedoch alles andere als „,naturwüchsiges“ oder „lebensweltliches“ Wissen. Der politische Habitus - die Wahrnehmungs- und Bewertungsschemata der Entscheidungsträger - sind zum Teil ,im Voraus“ inkorporiert worden: als der Bildungsminister noch Universitätslehrer war und der Gesundheitsminister noch Arzt, und zwar kein Laie, bloß noch kein Politiker. (Laienwissen wird unter bestimmten Bedingungen auch mobilisiert - aber darauf gehe ich jetzt nicht ein.)

Von den verschiedenen Wissensformen werden im Kontext eines noch weitgehend unausdifferenzierten sozialen Systems, wie Ungarn, ${ }^{14}$ diejenigen die wichtigste Rolle spielen, die ein autonomes Feld der Expertise eben nicht voraussetzen. Wenn es an Daten oder Experten, und auch an charismatischen Entscheidungsträgern im oben erwähnten Sinne mangelt, verstehen und benehmen sich gerne Entscheidungsträger selber wie Experten.

Zwei Prototypen des Expertenpolitikers gibt es. Der eine wird Fachpolitiker genannt. Der Begriff „Fachpolitiker“ existiert zwar auf Ungarisch und auf Deutsch, aber nicht zum Beispiel auf Französisch. Ein Fachpolitiker beschäftigt sich mit Fachfragen weil er / sie aufgrund seines / ihres Diplomabschlusses oder seiner / ihrer beruflichen Erfahrungen als fachkompetent gilt. Das sind zum Beispiel - in Ungarn - die Mitglieder der Gesundheitskommission des Parlaments. Der erste Prototyp ist also eher in der legislativen Branche tätig. Der andere ist der klassische Entscheidungsträger, der in der Regierung sitzt. Dieser „Experten-Entscheidungsträger“ (Staatssekretär, Minister etc.) positioniert sich jedoch fachkompetent und nichtpolitisch (apolitisch), und zwar aufgrund seiner inkorporierten Erfahrungen als Arzt und Krankenhausdirektor bzw. als Lehrer oder immer noch tätiger Universitätsprofessor.

Das sind natürlich nur Idealtypen. Um sie sofort ein bisschen in Frage zu stellen, zitiere ich ein Beispiel über eine „Übergangsform“ zwischen dem „Experten-Entscheidungsträger“ und

14 Auf jeden Fall gibt es kein „Feld” der Consultants und Experten. 
dem „Fachpolitiker“. Eine ehemalige Staatssekretärin, die sich jetzt als Fachpolitikerin versteht:

„Es ist, denke ich, von Vorteil, dass ich erlebtes Wissen habe, und andererseits bringe ich als Abgeordnete vom Land auch anderes Wissen mit. Es ist wichtig, denke ich. Und es ist auch wichtig, dass ich bei der Beziehung mit Angestellten im Gesundheitssystem dieselbe Kommunikationssprache beherrsche. Ich kann also viele verschieden Rollen verkörpern. Am meisten Nutzen bei der Konfliktlösung und Mediation habe ich von meiner Psychiaterausbildung. “

Diese beiden Untertypen des Fachpolitikers sind voneinander also nicht vollständig zu trennen, sie unterscheiden sich dennoch ganz klar vom charismatischen Typ der politischen Wissensdarstellung bzw. -anwendung. In allen Fällen handelt es sich um Erfahrungen, die im politischen Bereich als Formen des Wissens dargestellt und benutzt werden; und es handelt sich um einen politischen Habitus, dessen konstituierendes Element vorpolitische Erfahrung und außerpolitisches Wissen sind. Die von diesen beiden Typen von Fachpolitikern aufgeführten Diskurse werden als sachorientiert dargestellt, sie widersprechen also dem legal-rationalen Legitimationstyp nicht, während der / die charismatisch handelnde Fachpolitiker(in) Hingabe verspricht, Visionen offenbart und sich selber persönlich einsetzt.

Ich möchte hier das Interview mit einem sehr hochrangigen ungarischen Entscheidungsträger länger zitieren, weil es zeigt, wie die eigene Erfahrung als „Evidenz“ und „Expertenwissen“ konstruiert und benutzt wird, um den Mangel an harten, ,klassischen“, sicheren formellen Daten (z.B. Statistiken) zu überbrücken:

Nehmen wir ein konkretes Beispiel: Die nosokomialen Infektionen, also die Infektionen, die im Krankenhaus erworben wurden. Gibt es diesbezüglich Datenerhebungen?[...] Ja, aber im Rahmen des nationalen Statistischen Datenerhebungsprogrammes, die Daten kommen nicht zu uns [in die staatliche Krankenkasse].

Kommt es nicht vor, dass die staatliche Krankenkasse irgendwelche Konsequenzen daraus zieht, z. B. dass es in irgendeinem Krankenhaus zu viele gibt, und deshalb [...]?

Ich persönlich glaube nicht an die Daten, weil ich selber Arzt war. Ich war damals Chirurg und diese nosokomiale Geschichte war eine ziemlich wichtige Angelegenheit. Als ich damals noch dort angestellt war, haben wir den nosokomialen Bericht so gemacht, dass wir das Statistische Jahrbuch des letzten Jahres genommen haben und den nationalen Wert, sagen wir 1.3, herausgesucht haben, und dann hat der abteilungsleitende Chefarzt entschieden, dass es bei uns 1.32 oder 1.26 sein wird und diesem Sinne haben wir dann die Daten produziert. Wir können aus den Berichten aber [die Daten] herausfischen. Z.B. sind die Daten über die Komplikationen in den Daten über die stationären Aufenthalte integriert, also in dem Fall, dass das Krankenhaus korrekt Bericht erstattet, können wir diese aufarbeiten. Ich sage jetzt nicht zufällig ,, in dem Fall “. [...] Man kann diese klarerweise mit den verschiedenen Sterbedaten zusammenführen. In den Sterbedaten gibt es keine Fehler, das hat nichts mit Finanzierung zu tun, also kann man in diesem Sinne die Dinge filtern. [...] Wir sehen sehr wohl, dass wenn wir die Finanzierung der Therapie der einzelnen Krankheiten ändern, also wenn wir im Falle einiger Krankheiten mehr Geld geben, dann steigt im Allgemeinen die Anzahl dieser Krankheiten. Wir schauen dann, wo es im Vergleich eine Reduzierung gibt. So können wir abschätzen, was im Vergleich zu früher anders kodiert wird. Dann können wir den Trend anschauen, wie viel es von dem Alten in den letzten $X$ Jahren gab, und wenn wir annehmen, dass wir eine gute Regressionskurve haben, dann können wir sagen, heute ist es genau so. Und so können wir wieder alles herausfiltern. Das sind teils Tricks, teils statistische Methoden... 
Da sieht man wie in spezifischen Nichtwissensituationen das fehlende Wissen durch Erfahrungswissen substituiert (,,ich weiß ja wie es läuft, ich war selber Arzt"), als sicheres, oder gar sichereres Wissen dargestellt, oft auch inszeniert wird, dadurch wahrheitsfähig wirkt und als Basis für politisches Handel gebraucht werden kann.

Wissen als Erfahrung der Machthaber selber ist viel mehr als eine vage Erinnerung an Assistenzarztzeiten oder eine instinktive Vorangehensweise. Es ist eine Mischung von Neuaufarbeitung der doch zur Verfügung stehenden Daten, vom Einrechnen der statistischen Verzerrung, und von der Verwendung anderer, normalerweise nicht dazugehörender Daten. In diesem Beispiel geht es - vor allem - darum, selbst erfahrene, oder sogar selbst praktizierte Datenmanipulationsweisen zu kennen und Daten mit Hilfe von Statistik in „Wahrheit“ zu verwandeln, nutzbar zu machen. So wird in einer bewussten Nichtwissenssituation das mangelnde Wissen aus anderen Quellen ergänzt. Diese Substitution kommt durch eine Verschiebung des Fokus zustande: statt (,falsche“ oder „fehlende“) Evidenz rückt Erfahrung vor.

\section{Stakeholdern als Ersatzwissensträger}

Auch Lobbygruppen, NGOs, Stiftungen, Ärztekammer, Patientenverbände etc., also die so genannten Stakeholder, treten oft mit dem unverhüllten Ziel einer Policy-Änderung auf. Auch deren Wissen wird in bestimmten Kontexten als Ersatzwissen benutzt. Viele Autoren behaupten, dass Stakeholder, besonders die Interessensgruppen selber, Teil des politischen Systems sind. Im ungarischen Beispielfall sind sie aber keine richtigen „Anspruchsberechtigten“: ihre Meinung wird nur in Ausnahmefällen (in bestimmten „Nichtwissen-Fällen“) betrachtet, wie eine Analyse der konsultativen Gremien im Bildungswesen gezeigt hat (Bajomi 2006).

Die Informationsströme zwischen Entscheidungsträgern und den von ihren Entscheidungen betroffenen Personen bzw. Gruppen ist routinemäßig einseitig: die „Betroffenen“ - z.B. im Gesundheitswesen die Patientenverbände, Krankenhäuserverbände - bekommen zwar die Gesetzesentwürfe, ihr Meinung zählt aber nicht oder wird von der Opposition, die eine Wissenslücke, ein spezifisches Nichtwissen identifiziert, als Ersatzwissen thematisiert (Eröss / Mund 2007).

In anderen Fällen kann es dazu kommen, dass die Entscheidungsträger ein Nichtwissensgefühl empfinden, und selber die Stakeholder als Ersatzwissensträger befragen, üblicherweise eher informell. Wie im folgenden Beispiel, wo ein leitender Versicherungsfirmenmanager, der an Verhandlungen über die mögliche Teilprivatisierung der staatlichen Krankenkasse teilgenommen hat, berichtet:

„,Wir sagten nicht, dass wir so anständig wären, nicht bestimmte Leute herauszupicken [d.h. bei der Kundensuche gesündere Bürger zu bevorzugen], sondern dass Bedingungen geschaffen werden müssen, dass das nicht in unserem Interesse liegt. Und die Versicherungen sagten die ganze Zeit [bei den Verhandlungen mit den staatlichen Entscheidungsträgern] - und ich schwöre, mit den ehrlichsten Absichten, und es hört sich blöd an, dass ein Vertreter der Finanz-Geier so was sagt-dass niemand ehrlich glauben kann, dass ich es nicht machen werde, nur weil man es mir verbietet. Wenn ich also dem Versicherungsvertreter sage, er soll rotbackige Menschen suchen, die gut sichtbar gesund sind, dann wird er solche suchen. Ich muss auch nicht niederschreiben, er reicht, ich sage es ihm. "

Das ist ein Beispiel, wo die „Kapitalisten“ den Politikern die Mechanismen des Kapitalismus erklären, mit anderen Worten: Wissen aus einem „fremden“ Feld oder System, hier aus der Wirtschaft, ins politische System transferieren, um politische Wissenslücken zu überbrücken.

Eine Anthropologie des Staates ist in der Lage, die Komplexität der Verknüpfungsmechanismen zwischen Wissen und Politik aufzuzeichnen (Gailey). Eine politische Wissensanthro- 
pologie (oder qualitative Wissenssoziologie) hat dasselbe Ziel, sie beschäftigt sich aber auch mit nicht-staatlichen Wissensproduzenten. Sie fokussiert dabei auch auf profanes bzw. „heruntergesunkenes“ Wissen, auf profane Wahrnehmungsschemata, untersucht Tagungen und Gremien, wo Stakeholder und Vertreter der Zivilgesellschaft zur Emergenz von dieser Art von „Nicht-Wissen“ beitragen und gleichzeitig (ihre) alternativen Wissensformen hinzuziehen.

\section{Schlussbemerkungen. Herrschaft und Legitimation}

Wenn die Vermessung der sozialen Welt - zwangsläufig - doch an das Unvermessbare stößt, erwacht eine Art Unbehagen. Die Antwort auf dieses Unbehagen - im Kontext der steigenden Anfrage aufEvidenz - ist die Verwendung von alternativen Wissensformen: Erfahrungs-, bzw. praktisches Wissen, verkörpert-charismatisches oder lokales Wissen usw.

Eine Soziologie der ,praktischen Vernunft“, i.e. der Translationen im Sinne von Latour (z.B. Latour 1988) zeigt, dass im Netzwerk der Translation von humanen und nicht-humanen Akteuren gleichzeitig Experten, Entscheidungsträger, Wissenschaftler sowie Daten, Forschungsmethoden und Argumente zusammen in einem sich immer verändernden Verhältnis stehen, sie definieren sich gegenseitig, machen einander überhaupt erst möglich. In diesen Netzwerken wird auch alternatives Wissen aus „Nicht-Wissen“ erzeugt. Implizite Wissenselemente (Polányi 1985: 10) können in den Mittelpunkt des Netzwerkes hineinragen. Mit qualitativen Methoden - die der verstehenden politischen Soziologie - ist das Sichtbarmachen aber möglich.

Ich habe in diesem Artikel versucht, eine politische Agnatologie zu skizzieren: das Nichtwissenskonzept mittels der politischen Soziologie zu definieren und einige Ersatzmechanismen zu zeigen, die aus dem anscheinenden Nichtwissen bzw. aus der spezifischen Ignoranz verschiedene Ersatzwissensformen - z. B. soziologisches, charismatisches, praktisches, lokales Wissen, aber auch andere heteronome Wissensformen - erzeugen.

Der Mangel an Wissen ist ein soziales und politisches Konstrukt. Er entsteht in zwei Fällen, die beide auf Legitimationsstörungen des politischen Systems zurückzuführen sind. Der eine Fall ist der der bürokratisch-legalen Herrschaft, die sich auf institutionalisiertes, formales, wissenschaftliches Wissen stützen möchte, es aber nicht findet. Ersatzwissen muss also dringend erzeugt werden, um diese Wissenslücken zu erfüllen. Da gibt es drei Möglichkeiten: wenn der Mangel als kontingent wahrgenommen wird, werden die traditionellen, systemeigenen Wissenserzeugungsmechanismen mobilisiert. Der Einsatz eines systemeigenen Wissens bedeutet, dass bürokratisches Wissen mobilisiert wird: es werden zum Beispiel gesetzliche Analogien, oder entsprechende Entscheidungen des Bundesverfassungsgerichtes etc. gesucht. Wenn dies nicht möglich ist, der Mangel aber als vorübergehend bzw. leicht zu überbrücken erscheint, wird eine besondere Art von Ersatzwissensform miteinbezogen: Wissenschaft und zwar typischerweise Sozialwissenschaft und die Experten als Wissenschaftsmakler. Wenn aber der Mangel als strukturell permanent und trotzdem mit legal-rationalen Methoden zu überbrücken erscheint, wird entweder lokales oder transnationales Ersatzwissen verwendet oder auch Wissensformen, die von Stakeholdern im weitesten Sinne (Zivilorganisationen und Sozialpartnern) erzeugt bzw. vertreten werden.

Der andere Fall ist, wenn es in einer modernen Gesellschaft an Legitimität legal-rationaler Natur, und an Wissen rational-formalisierter Natur mangelt. Dann wird an charismatische Legitimität appelliert, die durch eingekörperte Wissensformen verschiedener Art unterstützt werden. Zwei Arten davon wurden hier angesprochen. Einerseits das charismatische Wissen im engeren Sinne: das, was man erlebt hat, wobei der Körper, auch in der Öffentlichkeit, als Beweis und Legitimationsquelle fungiert. Andererseits das praktische Wissen, das durch die Praxis der Entscheidungsträger als außerpolitisches Wissen internalisiert worden ist und als politischer skill verwendet wird; die so entstehende oder konstruierte Kompetenz ist die der 
einzelnen Person und nimmt dadurch auch am Legitimationsverschiebungsprozess teil, der letztendlich nicht nur mangelndes Wissen durch Wissenssubstitutionsformen, sondern auch mangelnde rationale Legitimität durch charismatische Legitimität ersetzt.

Experten müssen Broker des Nichtwissens werden. Das wissen sie genau. Ob sie sich auf der lokalen bzw. transnationalen Ebene neu positionieren, an anderen Formen der Wissenssubstitution teilnehmen oder einfach Science Fiction schreiben (z.B. ,Impact Analysis“) bzw. die verschiedenen Szenarien kombinieren, hängt vom Kontext ab und muss empirisch studiert werden. In unserer mitteleuropäischen Skizze scheint die Hochstilisierung des eigenen Wissens der Entscheidungsträger zum authentischen Wissen die dominierende Form zu sein: die eingekörperte bzw. die charismatisch verkörperte Wissensform derjenigen, ,,die es aus eigener Erfahrung kennen“. Erfahrung statt Evidenz. Feuer und Wasser? Nicht im Geringsten. Schlussendlich heißt doch der Begriff „Erfahrung“ auf Griechisch: $\varepsilon \mu \pi \imath \varepsilon \varepsilon \alpha$ (empireia).

\section{Literatur}

Altrichter, Herbert / Thomas Brüsemeister / Jochen Wissinger (Hrsg.)(2007): Educational Governance. Handlungskoordination und Steuerung im Bildungssystem, Wiesbaden.

Arrowsmith, James / Keith Sisson / Paul Marginson (2004): What can 'benchmarking' offer the open method of co-ordination?, in: Journal of European Public Policy 11 / 2, S. 311-328 (18).

Bajomi, Iván / Eszter Berényi / Eszter Neumann (2007): The social and cognitive mapping of policy. Education sector in Hungary, abgerufen im November 2009 unter http://www.knowandpol.eu/filead$\mathrm{min} / \mathrm{KaP} /$ content/Scientific_reports/Orientation1/O1_Final_Report_Hungary_educ1.pdf.

Bajomi Iván (2006): Konfliktusok és konszenzusképzés az oktatásban [Konflikte und Konsensbildung im Schulwesen], Budapest.

Bechmann, Gotthard / Silke Beck (2003): Gesellschaft als Kontext von Forschung. Neue Formen der Produktion und Integration von Wissen. Klimamodellierung zwischen Wissenschaft und Politik, Karlsruhe: Forschungszentrum Karlsruhe.

Beck, Ulrich (1996): Wissen oder Nicht-Wissen?, in: Ulrich Beck / Anthony Giddens / Scott Lash (Hrsg.), Reflexive Modernisierung. Eine Kontroverse, Frankfurt / Main, S. 289-315.

Blendon, Robert J. / Minah Kim / John M. Benson (2001): The Public Versus The World Health Organization On Health System Performance, in: Health Affairs 20 / 3, S. 10-20.

Bourdieu, Pierre (2001): Science de la science et réflexivité, Raisons d'agir, Paris.

Csányi, Yvonne (2007): „Helyi adatgyüjtés a sajátos nevelési igényű gyermekek körében egy OECDprojekt keretében" [Lokale Datenerhebung über Schüler mit sonderpädagogischen Forderungsbedarf, im Rahmen eines OECD-Projektes], Új Pedagógiai Szemle 2007 / 07.

Delvaux, Bernard / Eric Mangez (Hrsg.)(2008): Literature reviews on knowledge and policy, abgerufen unter www.knowandpol.eu/fileadmin/KaP/content/Scientific_reports/Literature_review/Know_Pol_ literature_review.pdf.

Eröss, Gábor (2008 a): Mozaik-Magyarország felfedezése, avagy az esetlegesség uralma [Mosaik-Land Ungarn entdecken, oder die Herrschaft der Kontingenz], in: Ders., Iskolarend. Kiváltság és különbségtétel a közoktatásban [Schulordnung. Privileg und Differenzierung im ungarischen Schulwesen] (mit Eszter Berényi und Balázs Berkovits), Budapest, S. 27-76.

Eröss, Gábor (2008 b): A habitus színe és visszája. Az iskolajelentések megalkotásának antropológiája [Habitus von beiden Seiten. Anthropologie der Konstruktion von Schulbedeutung], in: Ders., Iskolarend. Kiváltság és különbségtétel a közoktatásban [Schulordnung. Privileg und Differenzierung im ungarischen Schulwesen] (mit Eszter Berényi und Balázs Berkovits), Budapest, S. 217-278.

Eröss, Gábor / Katalin Mund et al. (2007): Unhealthy data, competing sciences. A double report about (a) the Hungarian health care system in general, and (b) the field of special educational needs, abgerufen 
November 2009 unter http://www.knowandpol.eu/fileadmin/KaP/content/Scientific_reports/Orientation1/O1_Final_Report_Hungary_health.pdf.

Felouzis, Georges / Françoise Liot / Joëlle Perroton (2005): L'apartheid scolaire. Enquête sur la ségrégation ethnique dans les collèges, Paris.

Gailey, Christine Ward (1985): The state of the state in anthropology, in: Dialectical Anthropology 9, S. 65-89.

Geis, Anna (2008): Die Rolle von Wissen und Nichtwissen in informellen politischen Steuerungsprozessen, in: Gunnar Folke Schuppert / Andreas Voßkuhle (Hrsg.), Governance von und durch Wissen, Berlin, S. 151-172.

Gibbsons, Michael / Camille Limoges / Helga Nowotny / Simon Schwartzman / Peter Scott / Martin Trow (Hrsg.)(1994): The New Production of Knowledge: The Dynamics of Science and Research in Contemporary Societies, London.

Gieryn, Thomas F. (1995): Boundaries of Science, in: Sheila Jasanoff et al. (Hrsg.), Handbook of science and technology studies, Thousand Oaks / CA.

Havas Gábor (2009): De ki hiszi el ezt nekünk? [Wer wird uns das überhaupt glauben?], Interview von Dóra Ónody-Molnár, in: Népszabadság vom 27. Januar 2009.

Julien Grenet (2008): PISA: une enquête bancale? La vie des idées, abgerufen im Februar 2008 unter http:// www.laviedesidees.fr/PISA-une-enquete-bancale.html.

Gross, Matthias (2007): The Unknown in Process: Dynamic Connections of Ignorance, Non-Knowledge and Related Concepts, in: Current Sociology 55, S. 742-759.

Habermas, Jürgen (1973): Legitimationsprobleme im Spätkapitalismus, Frankfurt / Main.

Halász, Gábor (2007): From deconstruction to systemic reform: educational transformation in Hungary, in: Orbis Scholae Vol. 1 / 2, S. 45-79.

Heclo, H. (1974): Modern Social Politics in Britain and Sweden: From Relief to Income Maintenance, New Haven / CT.

Hirschauer, Stefan (2008): ,,Körper macht Wissen - für eine Somatisierung des Wissensbegriffs“. in: KarlSiegbert Rehberg (Hrsg.), Die Natur der Gesellschaft. Verhandlungen des 33. Kongresses der Deutschen Gesellschaft für Soziologie in Kassel 2006 (Teil 2), Frankfurt / New York.

Kertesi, Gábor / Gábor Kézdi (2006): Expected long-term budgetary benefits to Roma education in Hungary, Budapest: Roma Education Fund.

Kertesi, Gábor (2007): A közoktatás mérési-értékelési rendszerének és az iskolák elszámoltathatósági rendszerének tökéletesítése [Die Verbesserung der Mess- und Evaluationssysteme im Bildungswesen, und der Verantwortlichkeitssysteme der Schulen], abgerufen im November 2009 unter http://oktatas.mholnap.digitalnatives.hu/wiki/M\%C3 \%A9r\%C3\%A9s-\%C3 \%A9rt\%C3\%A9kel\%C3\%A9selsz $\% \mathrm{C} 3 \% \mathrm{~A} 1$ moltathat $\% \mathrm{C} 3 \% \mathrm{~B} 3 \mathrm{~s} \% \mathrm{C} 3 \% \mathrm{~A} 1 \mathrm{~g} /$ javaslatok.

Kuhlicke, Christian (2006): Wissen und Naturkatastrophen: einige Überlegungen zum Thema Nichtwissen und ein empirisches Beispiel, in: Karl-Siegbert Rehberg (Hrsg.), Die Natur der Gesellschaft. Verhandlungen des 33. Kongresses der Deutschen Gesellschaft für Soziologie in Kassel 2006 (Teil 2), Frankfurt / New York, S. 844-857.

Latour, Bruno (1988): Science in Action: How to Follow Scientists and Engineers through Society, Cambridge / Mass.

Law, John (2003): Making a Mess with Method, veröffentlicht beim Centre for Science Studies, der Lancaster University, abgerufen im November 2009 unter http://www.lancs.ac.uk/fass/sociology/papers/ law-making-a-mess-with-method.pdf.

Lipsky, Michael (1983): Street Level Bureaucracy, New York.

Luhmann, Niklas (1984): Soziale Systeme. Grundriß einer Allgemeinen Theorie, Frankfurt / Main.

Luhmann, Niklas (1992): Die Wissenschaft der Gesellschaft, Frankfurt / Main.

Marmot, Michael G (2004): Evidence based policy or policy based evidence? In: BMJ 328, S. 906-907. 
Merton, Robert K (1968 [1949]): Social Theory and Social Structure, erw. Auflage, New York.

Merton, Robert K (1987) Three Fragments from a Sociologist's Notebooks: Establishing the Phenomenon, Specified Ignorance, and Strategic Research Materials, in: Annual Review of Sociology 13, S. 1-28.

Nassehi, Armin (2008): Soziologie. Zehn einführende Vorlesungen, Wiesbaden.

Polányi, Michael (1985): Implizites Wissen, Frankfurt / Main.

Reichert, Dagmar / Patricia Fry / Claudia Heid / Ursina Steinemann (2000): Wissenschaft als Erfahrungswissen, Wiesbaden.

Schuppert, Gunnar Folke (2008): Governance durch Wissen. Überlegungen zum Verhältnis von Macht und Wissen aus governancetheoretischer Perspektive, in: Gunnar Folke Schuppert / Andreas Voßkuhle (Hrsg.), Governance von und durch Wissen, Berlin, S. 260-303.

Straßheim, Holger (2008): Die Governance des Wissens, in: Gunnar Folke Schuppert / Andreas Voßkuhle (Hrsg.), Governance von und durch Wissen, Berlin, S. 49-74.

Tamás, Pál (2005): Kutatási brókerek: Társadalomtudományi szakértés és az új tudáspolitika [Forschungsmakler: Sozialwissenschaftliche Expertise und die neue Wissenspolitik], in: Magyar Tudomány 11, abgerufen im November 2009 unter http://www.matud.iif.hu/05nov/10.html.

Turner, Stephen (2001): What is the Problem with Experts?, in: Social Studies of Scienc 31 / 1, S. 123-149.

Voßkuhle, Andreas (2008 a): Sachverständige Beratung des Staates als Governanceproblem, in: Sebastian Botzem / Jeanette Hofmann / Sigrid Quack / Gunnar Folke Schuppert / Holger Straßheim (Hrsg.), Governance als Prozeß, Baden-Baden.

Voßkuhle, Andreas (2008 b): Das Konzept des rationalen Staates, in: Gunnar Folke Schuppert / Andreas Voßkuhle (Hrsg.), Governance von und durch Wissen, Berlin, S. 13-32.

Wehling, Peter (2004): Weshalb weiß die Wissenschaft nicht, was sie nicht weiß?, in: Stefan Böschen / Peter Wehling (Hrsg.), Wissenschaft zwischen Folgenverantwortung und Nichtwissen, Wiesbaden, S. 35-105.

Wehling, Peter (2006): Im Schatten des Wissens? Perspektiven der Soziologie des Nichtwissens, Konstanz.

Weingart, Peter (2003): Wissenschaftssoziologie, Bielefeld.

Wulf, Christoph et al. (2006): Bildung im Ritual. Schule, Familie, Jugend, Medien, Wiesbaden.

Dr. Gábor Eröss Institut für Soziologie Ungarische Akademie der Wissenschaften

Úri utca 49

1014 Budapest egabor@socio.mta.hu 